\title{
Gliflozinas: más que antidiabéticos orales. Una breve revisión de la literatura
}

\author{
Dra. Verónica González ${ }^{1}$
}

\section{Resumen}

La diabetes mellitus, la insuficiencia cardíaca y la enfermedad renal crónica tienen alta prevalencia en la población. Asimismo, estas patologías están comprendidas en un "círculo vicioso" porque comparten mecanismos fisiopatológicos que predisponen a su coexistencia en un mismo paciente, incrementando significativamente el riesgo de eventos cardiovasculares. Recientemente se han agregado al arsenal terapéutico las gliflozinas, un grupo de fármacos con beneficios en las tres enfermedades mencionadas. Saber cómo se desarrolló la investigación con estos fármacos y sus mecanismos de acción es fundamental para optimizar el tratamiento de los pacientes.

Palabras clave: $\quad$ INSUFICIENCIA CARDÍACA

DIABETES MELLITUS

ENFERMEDAD RENAL CRÓNICA

GLIFLOZINAS

\section{Gliflozins: more than oral antidiabetics. A brief review of the literature}

\section{Summary}

Diabetes mellitus, heart failure, and chronic kidney disease are highly prevalent in the population. Likewise, these pathologies are included in a "vicious circle" because they share pathophysiological mechanisms that predispose to their coexistence in the same patient, significantly increasing the risk of cardiovascular events. Gliflozins, a group of drugs with benefits in the three mentioned pathologies, have recently been added to the therapeutic arsenal. Knowing how research with these drugs and its mechanisms of action is essential to optimize the treatment of patients.

Key words: $\quad$ HEART FAILURE

DIABETES MELLITUS

CRONIC KIDNEY DISEASE

GLIFLOZINS

\section{Gliflozinas: mais do que antidiabéticos orais. Uma breve revisão da literatura}

\section{Resumo}

Diabetes mellitus, insuficiência cardíaca e doença renal crônica são altamente prevalentes na população. Estas patologias fazem parte de um "círculo vicioso", compartilhando mecanismos fisiopatológicos que predispõem à coexistência no mesmo paciente, e aumentando significativamente o risco de eventos cardiovasculares. As gliflozinas, são un grupo de drogas com benefícios das três patologias citadas, foram adicionadas recentemente ao arsenal terapêutico. Saber como foram desenvolvidas as pesquisas com esses medicamentos e seus mecanismos de ação é essencial para otimizar o tratamento dos pacientes.

Palavras chave: $\quad$ INSUFICIÊNCIA CARDÍACA

DIABETES MELLITUS

DOENÇA RENAL CRÔNICA

GLIFLOZINAS

1. Centro de Investigación de Cirugía Cardíaca y Trasplante. Casa de Galicia. Correo electrónico: vgonza77@gmail.com Recibido Mar 22, 2021; aceptado May 7, 2021 


\section{Introducción}

La diabetes mellitus 2 (DM2) se caracteriza por disminución de la secreción y/o un aumento de la resistencia periférica a la insulina generando hiperglicemia. La hiperglicemia se asocia a complicaciones macro y microvasculares (enfermedad cardiovascular, nefropatía, retinopatía y neuropatía) que aumentan la morbimortalidad $^{(1)}$. La mortalidad cardiovascular (CV) en la población general ha disminuido en las últimas décadas; sin embargo, en los pacientes con DM2 este descenso ha sido menos marcado. Es posible que esto se deba a la existencia de mecanismos fisiopatológicos distintos a los que conocemos y tratamos actualmente. La asociación bidireccional entre DM2 e insuficiencia cardíaca (IC) está bien establecida. Los pacientes diabéticos tienen una probabilidad entre dos a tres veces más que la población general de desarrollar IC y, asimismo, entre 20\% y $30 \%$ de los pacientes con IC asocian DM2. Si bien la asociación entre DM2 y enfermedad arteriosclerótica está vinculada a hiperglicemia, daño endotelial y factores de riesgo en común como la hipertensión arterial (HTA) y la obesidad, también la DM2 aumenta el riesgo de enfermedad CV por mecanismos distintos a los vinculados a la aterosclerosis $^{(2)}$. Por otra parte, la DM2 y la HTA son los principales factores de riesgo para desarrollar enfermedad renal crónica (ERC) y también predisponen al desarrollo de IC. A su vez, hasta un 50\% de los pacientes con IC y fracción de eyección del ventrículo izquierdo (FEVI) reducida presentan deterioro de las cifras de función renal, siendo esto un factor pronóstico adverso(3). Si bien la hiperglicemia se asocia con desarrollo de enfermedad macrovascular, los estudios que valoraron el control estricto de la glicemia y su relación con los eventos CV han mostrado resultados discordantes (UKPDS, ADVANCE, VADT, ACCORD ${ }^{(4-7)}$.

\section{Metodología de los ensayos con fármacos antidiabéticos}

A partir del año 2011 y debido a los resultados adversos reportados por la rosiglitazona a nivel CV, y en especial a la publicación de Nissen y colaboradores en el año $2009^{(8)}$, las agencias reguladoras de comercialización de medicamentos norteamericana y europea (Food and Drug Administration y la Agencia Europea de Medicamentos) publicaron guías con requisitos específicos para la aprobación de nuevos antidiabéti$\cos ^{(9)}$. En ellas se incluyen aspectos de eficacia y seguridad como el número de pacientes de los ensayos clínicos, su duración y la inclusión de población de mayor riesgo CV. Esto es así porque la probabilidad de presentar un evento adverso depende del tamaño de la muestra, del tiempo de seguimiento y del riesgo de la población a desarrollar dicho evento. Se consideró entonces pertinente aprobar estudios de menor tamaño poblacional y tiempo, pero que incluyan a pacientes de mayor riesgo CV. Finalmente, otro aspecto a considerar del diseño de los ensayos es que, con la mejora en los tratamientos $\mathrm{CV}$, es difícil diseñar estudios que se centren en una única variable como mortalidad $\mathrm{CV}$, infarto o internación por IC que requerirían grandes tamaños poblacionales o seguimientos a largo plazo. Debido a esto, los objetivos primarios suelen ser combinados, haciendo más dificultoso el análisis posterior, dado que a veces el objetivo se cumple a expensas, por ejemplo, de una de tres variables consideradas en conjunto.

\section{Inhibidores de los SGLT2}

La glucosa ingresa a las células a través de su unión a dos tipos de receptores de membrana: los transportadores GLUT (glucose transporters) y SGLT (sodium-glucose transporters). Los SGLT son cotransportadores de sodio y glucosa ( $\mathrm{Na} /$ glucosa) y se subdividen en dos isoformas: SGLT1 y SGLT2. El subtipo 1 está presente en el intestino y en el riñón a nivel del segmento S3 del túbulo contorneado proximal (TCP), donde se reabsorbe el $10 \%$ de la glucosa filtrada. Tiene gran afinidad por la glucosa, pero menos capacidad de transporte (1:1 con el Na). El SGLT2 está presente en riñón a nivel del segmento S1 y S2 del TCP, reabsorbe el $90 \%$ de la glucosa filtrada y tiene menos afinidad por la misma, pero mayor capacidad de transporte $(2 \mathrm{Glucosa}: 1 \mathrm{Na})^{(10)}$. Ambos cotransportadores son "secundariamente activos", es decir, no consumen energía porque transportan las moléculas por diferencia de concentración, pero necesitan antes la acción de la bomba de sodio y potasio $(\mathrm{Na} / \mathrm{K})$ que consume ATP. Los inhibidores de los SGLT2 (iSGLT2) provocan entonces diuresis osmótica al eliminar agua junto a glucosa y sodio(11,12).

Los estudios iniciales con iSGLT2 en animales comienzan en la década de 1990. Hasta ese momento la búsqueda de fármacos que mejoraran la hiperglicemia se basaba en moléculas capaces de estimular la secreción de insulina o de aumentar su sensibilidad a nivel periférico (figura 1).

La glucosa filtrada a nivel renal es absorbida en el túbulo proximal a través de los SGLT2 (cotrasportadores $\mathrm{Na}$ /Glucosa). Su inhibición por los iSGLT2 (inhibidores de los SGLT2) provoca la excreción de glucosa y sodio.

Ensayos con iSGLT2 en pacientes diabéticos Los estudios pioneros con estos fármacos en pacientes con DM2 son cuatro y se realizaron entre los 


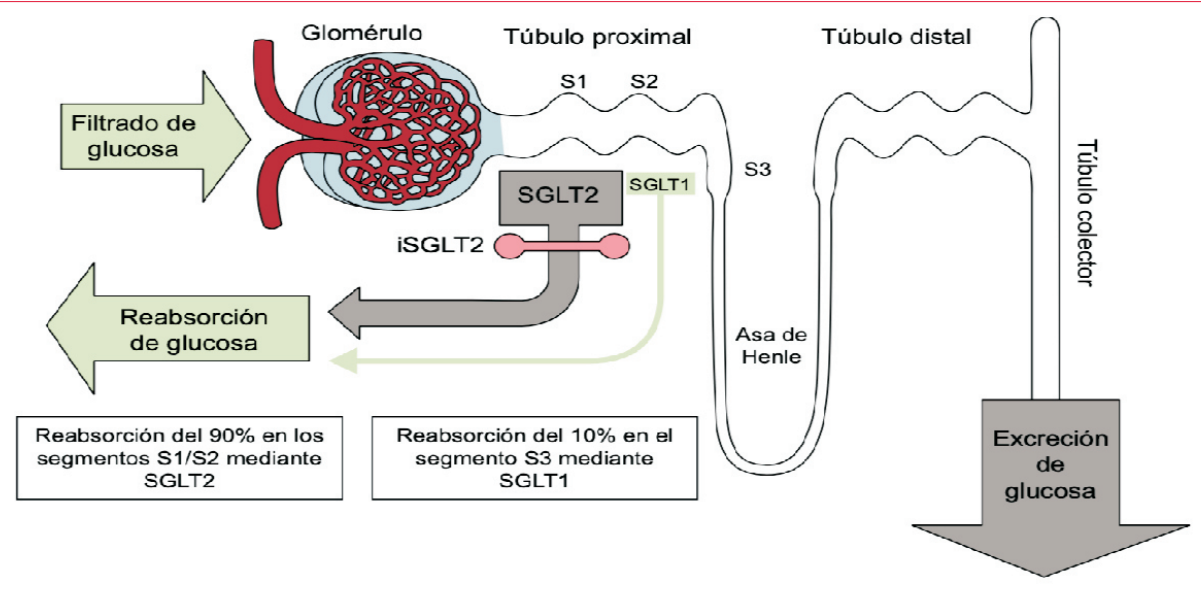

Figura 1. Mecanismo de acción iSGLT2.

años 2015 y 2019. El Empa Reg Outcome ${ }^{(13)}$ incluyó 7.020 pacientes con enfermedad CV establecida (ECVe) y los randomizó a recibir empagliflozina versus placebo. El objetivo primario combinado de muerte CV, infarto o stroke y los objetivos secundarios mortalidad global y hospitalizaciones por IC fueron estadísticamente significativos a favor del grupo tratamiento.

El Programa Canvas ${ }^{(14)}$ incluyó dos ensayos con criterios de inclusión y objetivos similares. Aleatorizó 10.000 pacientes con ECVe o alto riesgo CV a recibir canagliflozina versus placebo. El objetivo primario combinado fue el mismo que el Empa Reg Outcome y también fue estadísticamente significativo a favor del grupo canagliflozina, así como en la disminución de las hospitalizaciones por IC, de la albuminuria y de los eventos renales.

El estudio Credence ${ }^{(15)}$ incluyó 4.400 pacientes diabéticos, con ERC y macroalbuminuria, que no estuvieran recibiendo inhibidores de la enzima convertidora de angiotensina (IECA) o antagonistas de los receptores de angiotensina II (ARAII), randomizados a canagliflozina versus placebo. También mostró una reducción de las hospitalizaciones por IC y eventos renales.

El cuarto estudio es el Declare Timi $58^{(16)}$ con 17.000 pacientes randomizados a dapagliflozina versus placebo. Si bien alcanzó el objetivo de no inferioridad y no el de superioridad, mostró una disminución de las hospitalizaciones por IC. Este es el único de los cuatro estudios mencionados que incluye pacientes con IC definida, incluyendo $8 \%$ con FEVI reducida y $4 \%$ con FEVI preservada.

Un metaanálisis publicado por Zelniker y colaboradores $^{(17)}$ en el año 2019 que incluyó el Empa Reg Outcome, el Programa Canvas y el Declare-Timi 58, con 34.222 pacientes con DM2, 60\% con ECVe, $20 \%$ con ERC y $12 \%$ con IC y con seguimien- to promedio de 3,2 años, concluyó que los iSGLT2 disminuyeron el objetivo combinado de muerte $\mathrm{CV}$, infarto/stroke $11 \%$ (exclusivamente en el subgrupo de pacientes con ECVe), muerte CV u hospitalizaciones por IC $23 \%$ y $45 \%$ la progresión de la enfermedad renal; estos últimos dos objetivos independientemente de la presencia de ECVe.

El metaanálisis de Clare Arnott(18), que incluyó a los cuatro estudios descritos, con un total de 38.723 pacientes, de los cuales el 59\% presentaba ECVe, 20\% ERC y 10\% IC, concluyó que el tratamiento con iSGLT2 disminuyó la mortalidad CV, el infarto o el stroke no fatal, independientemente de la presencia o no de ECVe o IC. Los subgrupos analizados mostraron disminución de las hospitalizaciones por IC y de la mortalidad global.

Recientemente fue publicado un quinto trabajo en pacientes con DM2 con una cuarta gliflozina, la ertugliflozina, el Vertis $\mathrm{CV}^{(19)}$. Este estudio enroló 8.246 pacientes a ertugliflozina versus placebo con un seguimiento a 3,5 años. El diseño fue similar al de los otros ensayos con un objetivo primario de no inferioridad combinado de mortalidad CV, infarto o stroke y un objetivo secundario también combinado de muerte CV u hospitalización por IC. El objetivo primario y el secundario no mostraron superioridad de la ertugliflozina con respecto al placebo, pero sí se observó disminución de las hospitalizaciones por IC. Los autores en la discusión manejan dos hipótesis para explicar las diferencias en los resultados respecto a las otras gliflozinas: la mejoría del tratamiento farmacológico basal de los pacientes enrolados o la ausencia de un efecto de clase.

Posteriormente a la publicación del Vertis CV, McGuire ${ }^{(20)}$ y colaboradores publican un metaanálisis que incluye a los cinco trabajos publicados con iSGLT2 versus placebo en pacientes con DM2 (tabla 1). Incluye 46.969 pacientes, de los cuales el $66 \%$ pre- 


\begin{tabular}{|c|c|c|c|c|c|}
\hline & $\begin{array}{c}\text { EMPA-REG } \\
\text { OUTCOME }\end{array}$ & CANVAS & DECLARE-TIMI 58 & CREDENCE & VERTIS \\
\hline Año & 2015 & 2017 & 2019 & 2019 & 2020 \\
\hline Agente SGLT2i & Empagliflozina & Canagliflozina & Dapagliflozina & Canagliflozina & Ertugliflozina \\
\hline Participantes (N) & 7.028 & 10.142 & 17,16 & 4.401 & 8.246 \\
\hline Grupo SGLT2i & 4.687 & 5.795 & 8.582 & 2.202 & 5.499 \\
\hline Grupo placebo $(\mathrm{N})$ & 2.333 & 4.347 & 8.578 & 2.199 & 2.747 \\
\hline Edad, años (promedio \pm DS) & $63(9)$ & $63(8)$ & $64(7)$ & $63(9)$ & $64(8)$ \\
\hline Mujeres (\%) & 29 & 36 & 37 & 34 & 30 \\
\hline Diabetes $(\%)$ & 100 & 100 & 100 & 100 & 100 \\
\hline Paciente con IC (\%) & 10 & 14 & 10 & 15 & 24 \\
\hline Seguimiento (años) & 3,1 & 2,4 & 4,2 & 2,6 & 3 \\
\hline MACE HR (95\% IC) & $\mathbf{0 , 8 6}(0,75-0,97)$ & $\mathbf{0 , 8 6}(0,75-0,97)$ & $\mathbf{0 , 9 3}(0,84-1,03)$ & $\mathbf{0 , 8 0}(0,67-0,95)$ & $\mathbf{0 , 9 7}(0,85-1,11)$ \\
\hline Muerte CV HR (95\% IC) & $\mathbf{0 , 6 2}(0,49-0,77)$ & $\mathbf{0 , 8 7}(0,72-1,06)$ & $\mathbf{0 , 9 8}(0,82-1,17)$ & $\mathbf{0 , 7 8}(0,61-1,00)$ & $\mathbf{0 , 9 2}(0,77-1,03)$ \\
\hline HxIC HR (95\% IC) & $\mathbf{0 , 6 5}(0,50-0,85)$ & $\mathbf{0 , 6 7}(0,52-0,87)$ & $\mathbf{0 , 7 3}(0,51-0,88)$ & $\mathbf{0 , 6 1}(0,47-0,80)$ & $\mathbf{0 , 7 0}(0,54-0,90)$ \\
\hline Mcv / HxIC HR (95\% IC) & $\mathbf{0 , 6 6}(0,55-0,79)$ & $\mathbf{0 , 7 8}(0,67-0,91)$ & $\mathbf{0 , 8 3}(0,73-0,95)$ & $\mathbf{0 , 6 9}(0,57-0,83)$ & $\mathbf{0 , 8 8}(0,75-1-03)$ \\
\hline
\end{tabular}

senta ECVe, una edad promedio de 64 años y $66 \%$ de hombres. Entre las conclusiones, encuentran que los iSGLT2 reducen los eventos CV adversos mayores o MACE en $10 \%$, la mortalidad CV o la hospitalización por IC $22 \%$ y los eventos renales $38 \%$, no habiendo diferencias en cuanto a la presencia de ECVe. Se destaca, sin embargo, que la mortalidad CV presenta un grado elevado de heterogeneidad entre los estudios.

El metaanálisis de Neuen y colaboradores ${ }^{(21)}$, que analiza los cuatro primeros trabajos en pacientes con DM2, y que centra su análisis en la prevención de la falla renal, determina que los iSGLT2 disminuyen en $33 \%$ el riesgo de diálisis, trasplante o muerte de causa renal comparados con placebo. También concluye que disminuye el riesgo de injuria renal aguda en $25 \%$. Es importante señalar que el beneficio se mantiene aún en pacientes con filtrado glomerular (FG) $<45 \mathrm{~mL} / \mathrm{min} / 1,73 \mathrm{~m}^{2}$.

Estos efectos beneficiosos observados a nivel CV con una molécula definida como hipoglicemiante, sorprendieron a los investigadores y motivaron la puesta en marcha de estudios centrados en pacientes con IC, relegando a un segundo plano la condición de diabéticos.

\section{Ensayos en pacientes con insuficiencia cardíaca}

A finales del 2019 se publicó el primer estudio que valora la dapagliflozina en pacientes con IC con FEVI reducida: DAPA-HF ${ }^{(22)}$. Este estudio incluyó 4.744 pacientes con FEVI $\leq 40 \%$ y $\mathrm{FG}>30$
$\mathrm{mL} / \mathrm{min} / \mathrm{m}^{2}$, de los cuales $42 \%$ tenía DM2 y los randomizó a dapagliflozina $10 \mathrm{mg}$ vs placebo. El objetivo primario fue empeoramiento de la IC o mortalidad CV. En un seguimiento a 18 meses mostró $26 \%$ de reducción del objetivo primario en el grupo dapagliflozina, así como $30 \%$ y 18\%, respectivamente, en cada una de las variables que lo integran.

En el 2020 se publicaron los resultados del estudio con empagliflozina en pacientes con IC, el Emperor Reduced $^{(23)}$. Este estudio tiene un diseño similar al del DAPA-HF, pero agrega como objetivo secundario el empeoramiento del FG. El Emperor Reduced enroló pacientes con FEVI promedio menor a los del DAPA-HF (27\% vs 31\%), mayor valor de NT-proBNP basal (1.907 vs $1.437 \mathrm{pg} / \mathrm{mL}$ ), más pacientes tratados con inhibidores de la neprilisina (19\% vs $10 \%$ ) y con terapia de resincronización cardíaca (11\% vs $7 \%$ ). El seguimiento a 16 meses mostró $25 \%$ de reducción del objetivo primario a expensas de la hospitalización por IC, así como una reducción del 50\% del riesgo relativo de deterioro del FG. No fue estadísticamente significativa la reducción de la mortalidad CV ni la mortalidad global; pese a lo cual, la interpretación de la colectividad científica, en general, es que se trata de un efecto de clase.

\section{Ensayo en pacientes con enfermedad renal crónica}

Más recientemente se publicó el estudio DAPA $\mathrm{CKD}^{(24)}$, que analizó el beneficio de dapagliflozina contra placebo en pacientes con ERC (FG de 25 a 


\begin{tabular}{|c|c|c|c|c|c|c|c|c|}
\hline & $\begin{array}{c}\text { EMPA-REG } \\
\text { OUTCOME }\end{array}$ & $C A N V A S$ & $\begin{array}{c}\text { DECLARE } \\
\text { TIMI } 58\end{array}$ & CREDENCE & $D A P A-H F$ & $\begin{array}{c}\text { EMPEROR- } \\
\text { Reduced }\end{array}$ & VERTIS & $D A P A-C K D$ \\
\hline Año & 2015 & 2017 & 2019 & 2019 & 2019 & 2020 & 2020 & 2020 \\
\hline $\begin{array}{l}\text { Agente } \\
\text { SGLT2i }\end{array}$ & Empagliflozina & Canagliflozina & Dapagliflozina & Canagliflozina & Dapagliflozina & Empagliflozina & Ertugliflozina & Dapagliflozina \\
\hline $\begin{array}{l}\text { Participantes } \\
\text { (N) }\end{array}$ & 7.028 & 10.142 & 17.16 & 4.401 & 4.744 & 3.730 & 8.246 & 4304 \\
\hline Grupo SGLT2i & 4.687 & 5.795 & 8.582 & 2.202 & 2.373 & 1.863 & 5.499 & 2152 \\
\hline $\begin{array}{l}\text { Grupo placebo } \\
\text { (N) }\end{array}$ & 2.333 & 4.347 & 8.578 & 2.199 & 2.371 & 1.867 & 2.747 & 2152 \\
\hline $\begin{array}{l}\text { Edad, años } \\
\text { (promedio } \\
\pm \mathrm{DS} \text { ) }\end{array}$ & $63(9)$ & $63(8)$ & $64(7)$ & $63(9)$ & $66(11)$ & $67(11)$ & $64(8)$ & 62 \\
\hline Mujeres (\%) & 29 & 36 & 37 & 34 & 23 & 24 & 30 & 33 \\
\hline Diabetes (\%) & 100 & 100 & 100 & 100 & 42 & 50 & 100 & 68 \\
\hline $\begin{array}{l}\text { Paciente con IC } \\
(\%)\end{array}$ & 10 & 14 & 10 & 15 & 100 & 100 & 24 & 11 \\
\hline $\begin{array}{l}\text { Seguimiento } \\
\text { (años) }\end{array}$ & 3.1 & 2.4 & 4.2 & 2.6 & 1.5 & 1.3 & 3 & 2.4 \\
\hline $\begin{array}{l}\text { MACE HR } \\
\text { (IC 95\%) }\end{array}$ & $\begin{array}{c}\mathbf{0 , 8 6} \\
(0,75-0,97)\end{array}$ & $\begin{array}{c}\mathbf{0 , 8 6} \\
(0,75-0,97)\end{array}$ & $\begin{array}{c}\mathbf{0 , 9 3} \\
(0,84-1,03)\end{array}$ & $\begin{array}{c}\mathbf{0 , 8 0} \\
(0,67-0,95)\end{array}$ & & & $\begin{array}{c}\mathbf{0 , 9 7} \\
(0,85-1,11)\end{array}$ & \\
\hline $\begin{array}{l}\text { MUERTE CV } \\
\text { HR (IC 95\%) }\end{array}$ & $\begin{array}{c}\mathbf{0 , 6 2} \\
(0,49-0,77)\end{array}$ & $\begin{array}{c}\mathbf{0 , 4 7} \\
(0,72-1,06)\end{array}$ & $\begin{array}{c}\mathbf{0 , 9 8} \\
(0,82-1,17)\end{array}$ & $\begin{array}{c}\mathbf{0 , 7 8} \\
(0,61-1,00)\end{array}$ & $\begin{array}{c}\mathbf{0 , 8 2} \\
(0,69-0,98)\end{array}$ & $\begin{array}{c}\mathbf{0 , 9 2} \\
(0,75-1,12)\end{array}$ & $\begin{array}{c}\mathbf{0 , 9 2} \\
(0,77-1,03)\end{array}$ & $\begin{array}{c}\mathbf{0 , 8 1} \\
(0,58-1,12)\end{array}$ \\
\hline $\begin{array}{l}\text { HxIC HR } \\
\text { (IC 95\%) }\end{array}$ & $\begin{array}{c}\mathbf{0 , 6 5} \\
(0,50-0,85)\end{array}$ & $\begin{array}{c}\mathbf{0 , 6 7} \\
(0,52-0,87)\end{array}$ & $\begin{array}{c}\mathbf{0 , 7 3} \\
(0,61-0,88)\end{array}$ & $\begin{array}{c}\mathbf{0 , 6 1} \\
(0,47-0,80)\end{array}$ & $\begin{array}{c}\mathbf{0 , 7 0} \\
(0,59-0,83)\end{array}$ & $\begin{array}{c}\mathbf{0 , 6 9} \\
(0,59-0,86)\end{array}$ & $\begin{array}{c}\mathbf{0 , 7 0} \\
(0,54-0,90)\end{array}$ & \\
\hline $\begin{array}{l}\text { Mcv / HxIC } \\
\text { HR (IC 95\%) }\end{array}$ & $\begin{array}{c}\mathbf{0 , 6 6} \\
(0,55-0,79)\end{array}$ & $\begin{array}{c}\mathbf{0 , 7 8} \\
(0,67-0,91)\end{array}$ & $\begin{array}{c}\mathbf{0 , 8 3} \\
(0,73-0,95)\end{array}$ & $\begin{array}{c}\mathbf{0 , 6 9} \\
(0,57-0,83)\end{array}$ & $\begin{array}{c}\mathbf{0 , 7 5} \\
(0,65-0,85)\end{array}$ & $\begin{array}{c}\mathbf{0 , 7 5} \\
(0,65-0,86)\end{array}$ & $\begin{array}{c}\mathbf{0 , 8 9} \\
(0,75-1-03)\end{array}$ & $\begin{array}{c}\mathbf{0 , 7 1} \\
(0,55-0,92)\end{array}$ \\
\hline $\begin{array}{l}\text { Eventos renales } \\
\text { HR (IC 95\%) }\end{array}$ & $\begin{array}{c}\mathbf{0 , 5 4} \\
(0,40-0,75)\end{array}$ & $\begin{array}{c}\mathbf{0 , 6 0} \\
(0,47-0,77)\end{array}$ & $\begin{array}{c}\mathbf{0 , 5 3} \\
(0,43-0,66)\end{array}$ & $\begin{array}{c}\mathbf{0 , 7 0} \\
(0,59-0,82\end{array}$ & $\begin{array}{c}\mathbf{0 , 7 1} \\
(0,44-1,16)\end{array}$ & $\begin{array}{c}\mathbf{0 , 5 0} \\
(0,32-0,77)\end{array}$ & $\begin{array}{c}\mathbf{0 , 8 1} \\
(0,64-1,03)\end{array}$ & $\begin{array}{c}\mathbf{0 , 5 6} \\
(0,45-0,68)\end{array}$ \\
\hline
\end{tabular}

$75 \mathrm{~mL} / \mathrm{min} / 1,73 \mathrm{~m}^{2}$ y relación albuminuria/creatininuria entre 200 y 5.000). Para ser incluidos, los pacientes debían estar recibiendo IECA o ARAII. Se randomizaron 4.304 pacientes a dapagliflozina $10 \mathrm{mg}$ versus placebo, de los cuales $67 \%$ eran diabéticos y $10 \%$ tenía IC. El objetivo primario combinado fue deterioro del 50\% del FG, progresión de la ERC, mortalidad CV o mortalidad global. Se definió un objetivo secundario combinado de hospitalización por IC o mortalidad CV. El estudio fue suspendido prematuramente a los 2,4 años de seguimiento por el beneficio observado en el grupo tratamiento. El objetivo primario se redujo $43 \%$ en la rama tratamiento y el objetivo secundario lo hizo $29 \%$, independientemente de la presencia o no de DM2. Se trata, entonces, de la segunda molécula, luego de los ya probados IECA/ARAII, que muestra beneficios en los pacientes con ERC. Se encuentra en curso el estudio Empa Kidney que valora el efecto de la empagliflozina en pacientes con ERC.
Metaanálisis de ocho ensayos

Finalmente, un metaanálisis publicado recientemente que incluye a los ocho estudios mencionados, con 59.747 pacientes y evalúa el grado de heterogeneidad entre los distintos trabajos y poblaciones concluye que los iSGLT2 reducen la mortalidad global en 16\%, la mortalidad CV 16\%, la mortalidad CV y las hospitalizaciones por IC 31\%, el riesgo de infarto no fatal $9 \%$ y los eventos renales combinados $38 \%$, independientemente de la presencia de DM2, IC y del FG basal ${ }^{(25)}$. De las variables analizadas en estos ensayos hay dos que se destacan por sus resultados unánimemente favorables: las hospitalizaciones por IC y los eventos renales (tabla 2 ).

\section{Evidencia del uso de los isGLT2 en pacientes con DM2 en la vida real}

En el año 2018 se publicó el registro CVD Real(26,27) que comparó iSGLT2 versus otros hipoglucemiantes. Incluyó 300.000 pacientes de seis países. El 53\% recibía canagliflozina, $42 \%$ dapagliflozina y $5 \%$ em- 
pagliflozina. Se destaca que, a diferencia de los pacientes enrolados en los estudios randomizados, el $87 \%$ de los pacientes de este registro no presentaba ECVe. Las conclusiones del registro son: 39\% de reducción en hospitalizaciones por IC, $51 \%$ en mortalidad global y $46 \%$ en el objetivo combinado hospitalización por IC o muerte. Está claro que al tratarse de un registro hay que contemplar sus limitaciones.

\section{Mecanismo de acción de los iSGLT2}

Se ha señalado el mecanismo de acción básico de estas moléculas que originó su investigación como hipoglucemiantes (figura 1). Sin embargo, a la luz de los resultados obtenidos, se establece:

- el beneficio es independiente de la presencia o no de diabetes;

- el beneficio se mantiene aun con un FG reducido, donde la capacidad de reabsorber glucosa se encuentra disminuida;

- el cambio sobre la hemoglobina glicosilada (Hb1Ac) es marginal (-0,4\% promedio);

- la reducción en eventos CV se observa precozmente (en los primeros tres meses se separan las curvas), mientras que el beneficio de la reducción de la $\mathrm{Hb} 1 \mathrm{Ac}$ se manifiesta entre cinco y diez años luego del tratamiento;

- el escaso efecto en la reducción de eventos asociados a otros factores de riesgo CV clásicos

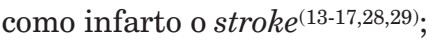

- y del beneficio marcado en reducción de la hospitalización por IC es que se empezaron a investigar mecanismos alternativos capaces de explicar la reducción de eventos con los iSGLT2.

En forma práctica podríamos dividir los mecanismos hasta ahora descritos en:

1) Efecto diurético o hemodinámico.

2) Efecto sobre el intercambiador $\mathrm{Na} / \mathrm{H}$.

3) Efectos metabólicos.

4) Efectos antiinflamatorios.

\section{1) Efecto diurético}

Esta hipótesis es la que ofrece menos discusión y se sustenta en el descenso ponderal de la presión arterial y de las hospitalizaciones por IC, observadas en forma unánime en los distintos estudios ${ }^{(30,31)}$. Asimismo, podría explicar el beneficio precoz del tratamiento con estos fármacos. Sin embargo, comparados con los diuréticos de asa, fármacos claves en el manejo de la IC, presentan algunas diferencias destacables.

El edema de los pacientes con IC suele ser intravascular e intersticial, siendo el intravascular el primero y más fácil de eliminar. Los iSGLT2 al provo- car glucosuria generan una diuresis osmótica que disminuye el volumen plasmático sin disminuir la osmolaridad plasmática. Esto ocasiona dos hechos destacados: el edema intersicial fluye hacia el espacio intravascular por gradiente de osmolaridad, pero al no existir una deplección significativa del espacio intravascular, no se estimula al sistema renina angiotensina aldosterona. A su vez, al aumentar la llegada de Na y volumen a la mácula densa, se estimula el feedback túbulo glomerular provocando vasoconstricción de la arteriola aferente, con la consiguiente disminución del hiperfiltrado glomerular y de la hipertensión glomerular. Esto provoca una disminución del daño renal que explica, entre otras cosas, el descenso de la albuminuria. Hay que destacar, sin embargo, que inicialmente provocan un deterioro del FG revertido posteriormente. De esta manera, el clearance intersticial es más marcado, mejorando la congestión con mínimo efecto sobre la presión arterial, el llenado arterial y la perfusión de los órganos, sin estimular la actividad neurohumoral y su ya conocido efecto deletéreo a largo plazo. Asimismo, si bien el aumento del hematocrito observado se explica por hemoconcentración, también se debe a que la disminución de la congestión renal disminuye el estrés parietal y la isquemia mejorando la función de las células yuxtaglomerulares encargadas de secretar eritropoyetina. Por último, al provocar glucosuria, generan disminución de la glucotoxicidad $^{(32-36)}$

\section{2) Intercambiador $\mathrm{Na} / \mathrm{H}$}

Si bien no está firmemente demostrado que los iSGLT2 inhiban a este intercambiador, se ha postulado que por la similitud morfológica del intercambiador $\mathrm{Na} / \mathrm{H}$ (NHE) con el del cotransportador Na-glucosa, estos fármacos podrían inhibir el primero ${ }^{(39)}$. Existen nueve isoformas del intercambiador NHE distribuidas en el organismo. A nivel cardíaco y de los vasos se encuentra el NHE1, que es el responsable de mantener el pH miocárdico y de evitar la lesión por isquemia reperfusión ${ }^{(40)}$. Las isoformas NHE2, 3 y 4 se encuentran en el riñón y son estimulados por mecanismos neuroendócrinos (como los que se hallan en la IC), por insulina y por glucosa (ambos aumentados en la DM2) generando una regulación en más de los mismos y el consiguiente aumento en la recaptación de $\mathrm{Na}$. Cuando existe isquemia como en la IC, ya sea por coronariopatía o por fibrosis, hay descenso del $\mathrm{pH}$ y una estimulación del NHE1 generando aumento del Na intracelular que posteriormente es intercambado por Ca. En última instancia, el aumento del Ca intracelular genera fibrosis e hipertrofia mediadas por la calcineurina. Este mecanismo se ha propuesto para ex- 
plicar la cardiotoxicidad, la nefrotoxicidad y la resistencia a los diuréticos en la IC(34-39). Recientemente se ha publicado un trabajo experimental realizado con miocitos de ratas que pone en duda la acción de la empagliflozina sobre el intercambiador NHE1. Serán necesarios nuevos ensayos para dilucidar este mecanismo ${ }^{(40)}$.

\section{3) Efectos metabólicos}

\section{a) Metabolismo cardíaco normal}

El miocardio obtiene energía de dos fuentes: el adenosín trifosfato (ATP) y la fosfocretina (PCr). Este último se utiliza cuando la producción de ATP no es suficiente. El músculo cardíaco tiene un alto recambio de ATP ( 6 a $37 \mathrm{~kg} /$ día), un flujo coronario de 200 $\mathrm{ml} / \mathrm{min}$ y un consumo de oxígeno $\left(\mathrm{O}_{2}\right)$ de los más altos del organismo (4,3 mmol $/ \mathrm{kg} / \mathrm{min})$. El $95 \%$ del ATP se obtiene a través de la fosforilación oxidativa y el 5\% restante de la glucólisis anaeróbica. El miocardio necesita gran cantidad de energía para satisfacer los requerimientos de las proteínas sarcoplasmáticas y para esto se vale de un principio llamado "flexibilidad de sustrato", que implica que puede cambiar de sustrato según la disponibilidad. En condiciones fisiológicas, el $70 \%$ de la energía se obtiene a partir de los ácidos grasos, 20\% de la glucosa y el $10 \%$ restante de los aminoácidos, el lactato y las cetonas. En condiciones en las que abundan los carbohidratos ("reserva") la glucosa es el principal sustrato, pero durante el ejercicio intenso o el ayuno prolongado, el lactato se transforma en la principal fuente de obtención de energía ${ }^{(41)}$.

\section{b) Metabolismo cardíaco en la insuficiencia cardíaca}

El miocardio normal sólo tiene reservas para 5 o 10 segundos, de modo que en presencia de isquemia o déficit de aporte de $\mathrm{O}_{2}$, rápidamente hace uso del ya mencionado mecanismo "flexibilidad de sustrato"(42). En este escenario se jerarquiza la glucólisis a través de la oxidación de la glucosa basado en el llamado ciclo de Randle, que postula que existe una competencia entre la oxidación de la glucosa y la de los ácidos grasos. Dado que en la IC existe isquemia y que la oxidación de la glucosa requiere menos $\mathrm{O}_{2}$, con una relación producción de $\mathrm{ATP} /$ consumo de $\mathrm{O}_{2}$ mayor que la de los ácidos grasos, 2,58 vs 2,33, la glucosa pasa a ser el sustrato preferido. Sin embargo, el rendimiento absoluto de moléculas de ATP con la glucosa es de 31 y con ácidos grasos 105. La perpetuación de esta situación, sumado al efecto tóxico de la glucosa, lleva al concepto de que "la insuficiencia cardíaca genera insuficiencia cardíaca"(41-44). Se produce así un descenso de la rela- ción fosfocretina/ATP que es un predictor de mortalidad en pacientes con IC dilatada ${ }^{(45)}$.

\section{c) Metabolismo cardíaco en la diabetes}

En los pacientes con diabetes e hiperglicemia hay un déficit de utilización de la glucosa, ya sea por déficit de insulina o por disminución de su sensibilidad. Esto lleva a una mayor utilización de los ácidos grasos, pero su consumo prolongado produce lipotoxidad y estrés oxidativo, lo que determina en el miocardio el desarrollo de hipertrofia, rigidez y falla diastólica; hallazgos habituales en la miocardiopatía diabética. Asimismo, la DM2 es el prototipo de abundancia de sustrato generando una pérdida del mecanismo "flexibilidad de sustrato".

\section{d) Metabolismo cardíaco en isquemia aguda}

Frente a la isquemia aguda se produce apoptosis e inflamación que conducen a un remodelado adverso, posterior hipertrofia y alteraciones del tejido conectivo, además de isquemia por reperfusión, que es otro mecanismo que contribuye al daño miocárdico. En este contexto, el metabolismo oxidativo de la glucosa y de los ácidos grasos se ve limitado, por lo que se produce una glucólisis anaerobia. Esta vía, además de ser energéticamente menos eficiente, genera lactato y protones que descienden el $\mathrm{pH}$ intracelular, responsable en última instancia de la disfunción contráctil.

\section{e) Metabolismo cardíaco en el ayuno prolongado}

Las cetonas son la fuente de energía más eficiente, pero sólo son producidas en situaciones de ayuno o "ayuno símil", como son el ejercicio intenso, las dietas cetogénicas o la diabetes con mal control y aumento de la lipólisis (obtención de ácidos grasos a partir de los triglicéridos). Las cetonas son el beta hidroxibutirato, la acetona y la acetoacetato, producidas a nivel hepático y liberadas al torrente sanguíneo. En situaciones normales en las que existen concentraciones elevadas de insulina y esta puede ser utilizada, la lipólisis y la cetogénesis se encuentran inhibidas; por el contrario, situaciones que provoquen una disminución de la insulina, estimulan la cetogénesis. Los órganos que más utilizan las cetonas como fuente de energía son el cerebro, el corazón y los riñones. La obtención de ATP a partir de cetonas, como el beta hidroxibutirato, es $31 \%$ más efectiva y $27 \%$ menos costosa que la obtenida a partir de los ácidos grasos. Los iSGLT2 al provocar glucosuria y generar una disminución de la insulina plasmática estimulan la glucogenólisis y la cetogénesis hepática, a la vez que disminuyen la aclaración de cetonas por el riñón generando un mecanis- 


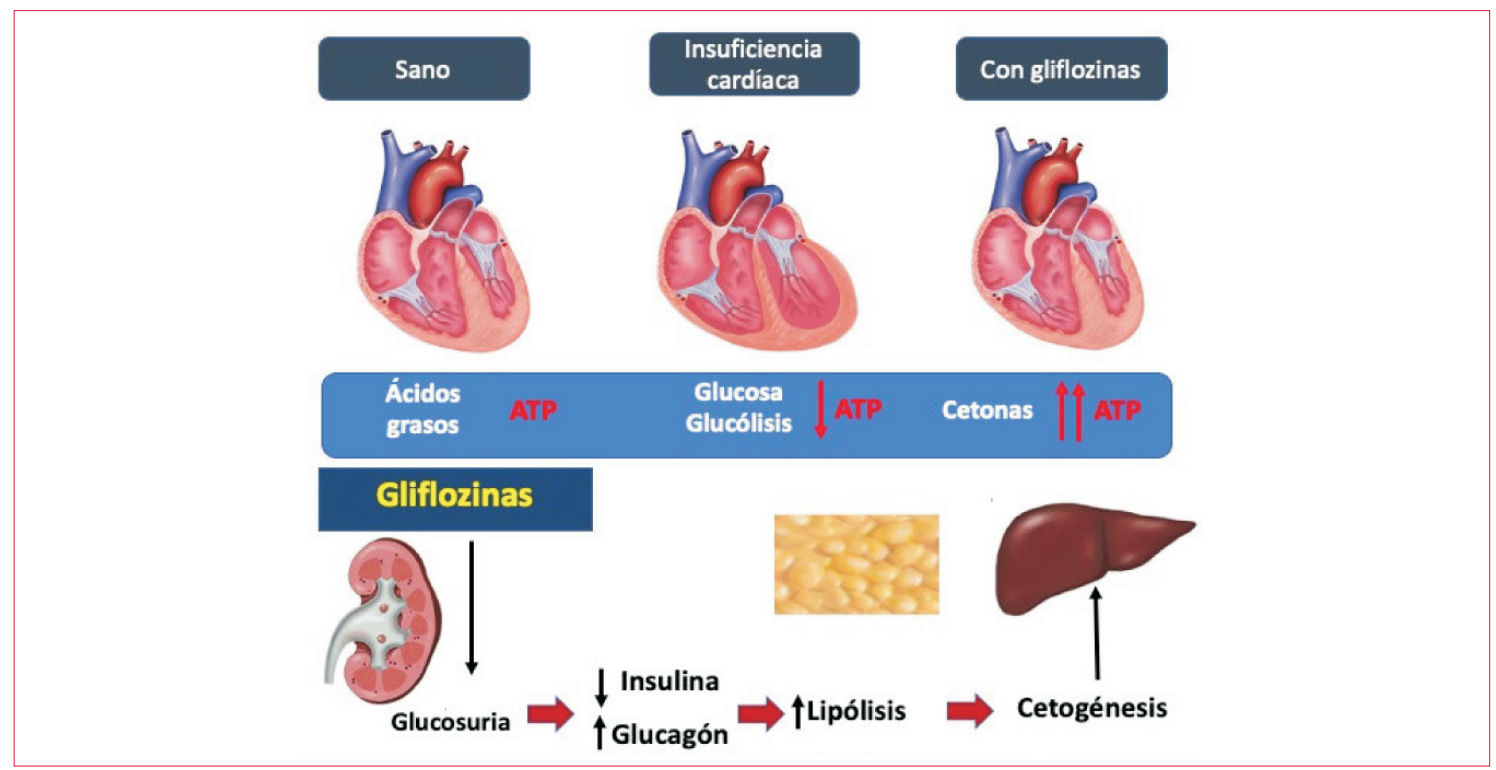

Figura 2. Efecto metabólico. Metabolismo energético en el corazón sano, en la insuficiencia cardíaca y con gliflozinas. ATP: adenosín trifosfato. Adaptado de Carlos G. Santos-Gallego MD ${ }^{(46)}$.

mo de obtención de energía más efectivo para el miocardio y el riñón.

A su vez, se postula que los iSGLT2, al provocar glucosuria, generan una pérdida calórica que simularía un estado de "ayuno prolongado", estimulando el cambio de fuente energética hacia el de las cetonas, que, como ya se ha dicho, es la más eficiente. Hay estudios preliminares que muestran que la empagliflozina estimula el consumo de cetonas $^{(46)}$.

Ya hay ensayos que valoran los efectos de las gliflozinas en el remodelado inverso ventricular. Recientemente se publicó el ensayo Empa Tropism ${ }^{(47)}$ que analiza con resonancia magnética cardíaca los cambios en los volúmenes ventriculares y la FEVI en pacientes randomizados a empagliflozina versus placebo. Los resultados demuestran cambios significativos en la rama tratamiento.

La disminución brusca de la glicemia provoca, además, disminución de la secreción de insulina y mejoría de la función de las células beta, así como un aumento del glucagón plasmático con aumento de la lipólisis y disminución de la lipotoxicidad (figura 2).

\section{3) Efectos antiinflamatorios}

Los programas celulares básicos son: crecimiento, reproducción y mantenimiento. Un ambiente favorable estimula un programa de crecimiento y reproducción, en tanto que uno hostil, de mantenimiento o sobrevida ${ }^{(48,49)}$. El ambiente hostil está representado por situaciones patológicas que van desde el estrés psicosocial o la escasez de alimentos, a la diabetes, la hiperglicemia y la IC que promueven progra- mas celulares alternativos como el de defensa o el de latencia.

El programa de defensa se caracteriza por un estímulo importante del sistema inmune y del metabolismo anabólico, con aumento de prooncogenes e interleukinas proinflamatorias. El estado inflamatorio forma parte de la fisiopatología de la IC, la diabetes y la enfermedad renal, pero su análisis excede el propósito de esta revisión. La mayor parte de estos mecanismos está mediada por la estimulación de la vía de la enzima mtOR y de la Akt.

El programa de latencia o "inanición símil”, por el contrario, se caracteriza por preservación de la energía y alta resistencia al estrés. Es un estado semejante al de la hibernación de algunos animales, donde se suprime el metabolismo anabólico y disminuye la secreción de insulina. Este mecanismo está mediado por dos enzimas: la AMPK (adenosín monofosfato kinasa) y SIRT1 (sirtuina). Su síntesis se encuentra estimulada no sólo en condiciones de ayuno, sino también cuando existe hipoxia, radicales libres, organelos o proteínas dañadas. La SIRT1 estimula la actividad antioxidante, disminuye la respuesta inflamatoria y la liberación de radicales libres. Por su parte, AMPK preserva la actividad mitocondrial y disminuye la respuesta proapoptótica. Ambas, disminuyen el anabolismo inhibiendo la gluconeogénesis y la lipogénesis, y promoviendo la oxidación de los ácidos grasos y la cetonemia ${ }^{(51-53)}$. Estas enzimas promueven, además, la autofagia. La autofagia es una vía de degradación lisosomal que se basa en la eliminación de los constituyentes dañados, reciclando componentes útiles para la síntesis de nuevas moléculas, lo que genera a la vez ATP 


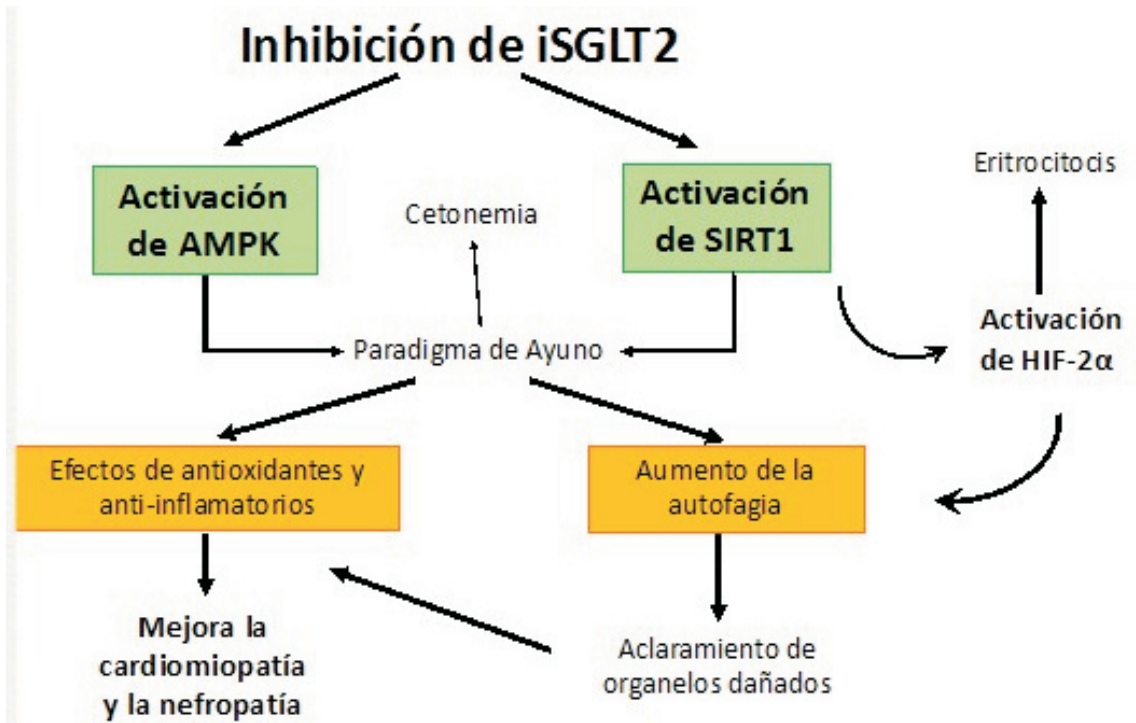

Figura 3. Mecanismo antiinflamatorio. Rol de los iSGLT2 (inhibidores de los SGLT2) en el mecanismo antiinflamatorio. AMPK: adenosín monofosfato kinasa; SIRT1: sirtuina 1.

(por ejemplo, a través de la degradación de glucógeno y lípidos) y preserva la homeostasis celular. La diabetes, por el contrario, disminuye la actividad de ambas enzimas, generando miocitos disfuncionantes y promoviendo la inflamación miocárdica.

Los iSGLT2 estimulan la producción de SIRT1y AMPK a la vez que disminuyen la de mTOR y Akt. Esto lleva a una disminución del estrés oxidativo, mejoría de la función mitocontrial, disminución de la inflamación, disminución del daño sobre la microvastulara coronaria, mejoría de la función contráctil y disminución de la cardio y de la nefropatía (figura 3). La evidencia clínica que sostiene esta teoría antiinflamatoria se fundamenta en tres elementos:

1. La glucosuria generada por los iSGLT2 produce pérdida de calorías, disminución de la gluconeogénesis y aumento de la oxidación los ácidos grasos y de las cetonas.

2. El aumento del hematocrito observado con los iSGLT2 sería secundario a la deprivación de $\mathrm{O}_{2}$ (secundario al estado "símil inanición") y estaría mediado por los factores inducibles por hipoxia HIF $2 \alpha$ y HIF $1 \alpha$.

3. La metformina también produce un aumento de SIRT1/AMPK y disminución de mTOR/Akt, pero no estimula HIF1 $\alpha$. Esto podría explicar el descenso leve del hematocrito observado con el uso de metformina.

Hay un aspecto adicional relevante de los iSGLT2 que es de relevancia en el beneficio renal y está vinculado a la disminución de la uricemia. La glucosa ingresa a las células a través de los SGLT y de los
GLUT, siendo estos últimos también los transportadores responsables de la reabsorción de los uratos. Al ser inhibidos los SGLT2 por las gliflozinas, la glucosa compite por los GLUT9B y esto resulta en una disminución de la reabsorción de uratos ${ }^{(42,54)}$. La hiperuricemia es un elemento característico de los pacientes con ERC y su tratamiento mejora el pronóstico.

\section{Indicaciones actuales para el uso de iSGLT2}

Insuficiencia cardíaca: a partir de la publicación de los estudios Dapa-HF y Emperor Reduced, la evidencia acerca del beneficio de estos fármacos en los pacientes con IC y FEVI reducida, independientemente de la presencia de DM2, es clara. Si bien es posible discutir la oportunidad de incluirlos en el esquema terapéutico de los cuatro "pilares" que han demostrado mejorar la morbimortalidad en los pacientes con IC y FEVI reducida, no quedan dudas sobre el beneficio de darlos ${ }^{(55)}$. Dapagliflozina y empagliflozina fueron aprobadas por la Food and Drug Administration (FDA) y la Agencia Europea de Medicamentos para este grupo de pacientes. Recientemente se dieron a conocer los resultados en fase III del estudio Emperor Preserved, que compara empagliflozina versus placebo en pacientes con FEVI mayor a $40 \%$ y muestra una reducción del objetivo primario combinado de muerte $\mathrm{CV} u$ hospitalizaciones por IC. Es necesario contar con la publicación del estudio para un análisis completo, pero sería la primer molécula en demostrar beneficio en puntos duros en pacientes con FEVI preservada. 
Diabetes mellitus 2: recientemente se publicó una actualización del tratamiento por parte de la Asociación Americana de Diabetes (ADA) y de la Sociedad Europea de Cardiología (ESC) ${ }^{(56)}$ que establece que en los pacientes diabéticos con ECVe o con alto riesgo deben recibir iSGLT2. Existen diferencias entre la ESC y la ADA con respecto a cuál de los fármacos, GLP-1 o iSGLT2, debería indicarse como primera línea.

Enfermedad renal crónica: la evidencia en cuanto a los beneficios en este grupo de pacientes es categórica en todos los trabajos en general y, en particular, en el Credence y el Dapa CKD, que incluyen pacientes con ERC avanzada(57,58). Recientemente, la FDA aprobó el uso de dapagliflozina en pacientes con ERC.

Verónica González,

https://orcid.org/0000-0002-8578-1517

\section{Bibliografía}

1. Gu K, Cowie CC, Harris MI. Diabetes and decline in heart disease mortality in US adults. JAMA. 1999;281(14):1291-7. doi: 10.1001/jama.281.14.1291

2. Tamargo J. Sodium-glucose cotransporter 2 inhibitors in heart failure: potential mechanisms of action, adverse effects and future developments. Eur Cardiol. 2019; 14(1):23-32. doi: 10.15420/ecr.2018.34.2 Fe de erratas en: Eur Cardiol.2019; 14(3):201.

3. Damman K, Valente MAE, Voors AA, O'Connor CM, Van Veldhuisen DJ, Hillege HL. Renal impairment, worsening renal function, and outcome in patients with heart failure: an updated meta-analysis. Eur Heart J. 2014;35(7):455-69. doi: 10.1093/eurheartj/eht386

4. Intensive blood-glucose control with sulphonylureas or insulin compared with conventional treatment and risk of complications in patients with type 2 diabetes, UKPDS 33. UK Prospective Diabetes Study (UKPDS) Group. Lancet. 1998;352:837-53. Fe de erratas en: Lancet 1999 Aug 14;354(9178):602.

5. Patel A, MacMahon S, Chalmers J, Neal B, Billot L, Woodward M, et al. Intensive blood glucose control and vascular outcomes in patients with type 2 diabetes. N Engl J Med. 2008;358(24):2560-72. doi: 10.1056/NEJMoa0802987

6. Duckworth W, Abraira C, Moritz T, Reda D, Emanuele N, Reaven PD, et al. Glucose control and vascular complications in veterans with type 2 diabetes. N Engl J Med. 2009;360(2) :129-39. doi: 10.1056/NEJMoa0808431

7. Ismail-Beigi F, Craven T, Banerji MA, Basile J, Calles J, Cohen RM, et al. Effect of intensive treatment of hyperglycemia on microvascular com- plications of type 2 diabetes: an analysis of the ACCORD randomized trial. Lancet. 2010;376(9739): 419-30. doi:10.1016/S0140-6736(10)60576-4

8. Nissen SE, Wolski K. Effect of rosiglitazone on the risk of myocardial infarction and death from cardiovascular causes. steven E. N Engl J Med. 2007;356 (24) :2457-71. doi:10.1056/NEJMoa072761. Fe de erratas en: N Engl J Med. 2007 ;357(1):100.

9. Ortega Basagoiti R. Peregrinaje de un antidiabeìtico hasta su aprobacioìn por las agencias internacionales del medicamento. Situacioìn actual. Av Diabetol. 2011;27(4):107-14. doi: 10.1016/j.avdiab.2011. 08.001

10. Lee WS, Kanai Y, Wells RG, Hediger MA. The high affinity na+/glucose cotransporter re-evaluation of function and distribution of expression. J Biol Chem. 1994;269(16):12032-39.

11. Kimura G. Importance of inhibitingssodium-glucose co-transporter and its compelling indication in type 2 diabetes: pathophysiological hypothesis. J Am Soc of Hypertens. 2016;10(3):271-8. doi: 10.1016/j. jash.2016.01.009

12. Wanner C. EMPA-REG OUTCOME: the nephrologist's point of view. Am J Cardiol. 2017;120 1Supl:S59-67. doi: 10.1016/j.amjcard.2017.05.012

13. Zinman B, Wanner C, Lachin JM, Fitchett D, Bluhmki E, Hantel S, et al. Empagliflozin, cardiovascular outcomes, and mortality in type 2 Diabetes. N Engl J Med. 2015;373(22):2117-28. doi: 10.1056/ NEJMoa1504720

14. Neal B, Perkovic V, Mahaffey KW, de Zeeuw D, Fulcher G, Erondu N, et al. Canagliflozin and cardiovascular and renal events in type 2 diabetes. $\mathrm{N}$ Engl J Med. 2017;377(7):644-57. doi: 10.1056/ NEJMoa1611925

15. Perkovic V, Jardine MJ, Neal B, Bompoint S, Heerspink HJL, Charytan DM, et al. Canagliflozin and renal outcomes in type 2 diabetes and nephropathy. N Engl J Med. 2019;380(24):2295-306. doi:10.1056/NEJMoa1811744

16. Wiviott SD, Raz I, Bonaca MP, Mosenzon O, Kato ET, Cahn A, et al. Dapagliflozin and cardiovascular outcomes in type 2 diabetes. N Engl J Med. 2019;380(4):347-57. doi: 10.1056/NEJMoa1812389

17. Zelniker TA, Wiviott SD, Raz I, Im K, Goodrich EL, Bonaca MP, et al. SGLT2 inhibitors for primary and secondary prevention of cardiovascular and renal outcomes in type 2 diabetes: a systematic review and meta-analysis of cardiovascular outcome trials. Lancet. 2019;393(10166):31-9. doi: 10.1016/ S0140-6736(18)32590-X

18. Arnott C, Li Q, Kang A, Neuen BL, Bompoint S, Lam CSP, Rodgers A, et al. Sodium-glucose cotransporter 2 inhibition for the prevention of cardiovascular events in patients with type 2 diabetes me- 
llitus: a systematic review and meta-analysis. J Am Heart Assoc. 2020;9:e014908.doi: 10.1161/JAHA. 119.014908

19. Cannon CP, Pratley R, Dagogo-Jack S, Mancuso J, Huyck S, Masiukiewicz U. Cardiovascular outcomes with Ertugliflozin in type 2 diabetes. N Engl J Med. 2020;383(15):1425-35. doi: 10.1056/ NEJMoa2004967

20. McGuire DK, Shih WJ, Cosentino F, Charbonnel B, Cherney DZI, Dagogo-Jack S, et al. Association of SGLT2 Inhibitors with cardiovascular and kidney outcomes in patients with type 2 diabetes: a meta-analysis. McGuire, Darren. JAMA Cardiol doi:10.1001/jamacardio.2020.4511

21. SGLT2 inhibitors for the prevention of kidney failure in patients with type 2 diabetes: a systematic review and meta-analysis. Lancet Diabetes Endocrinol. 2019;7(11):845-54. doi: 10.1016/S2213-8587(19)30256-6

22. Dapagliflozin in Patients with Heart Failure and Reduced Ejection Fraction J.J.V. McMurray, et al., for the DAPA-HF Trial Committees and Investigators. N Engl J Med. 2019;381(21):1995-2008. doi: 10.1056/ NEJMoa1911303

23. Packer M, Anker SD, Butler J, Filippatos G, Pocock SJ, Carson P, et al. Cardiovascular and Renal Outcomes with Empagliflozin in Heart Failure. N Engl J Med. 2020;383(15):1413-24. doi: 10 1056/NEJMoa2022190

24. Dapagliflozin in patients with chronic kidney disease. N Engl J Med. 2020;383(15):1436-46. doi: 10. 1056/NEJMoa2024816

25. Salah HM, Al'Aref SJ, Khan MS, Al-Hawwas M, Vallurupalli S, Mehta JL, et al. Effect of sodium-glucose cotransporter 2 inhibitors on cardiovascular and kidney outcomes-systematic review and meta-analysis of randomized placeboc-ontrolled trials. Am Heart J. 2021;232:10-22. doi: 10.1016/ j.ahj.2020.10.064

26. Kosiborod M, Cavender MA, Fu AZ, Wilding JP, Khunti K, Holl RW, et al. Lower risk of heart failure and death in patients initiated on sodium-glucose cotransporter-2 inhibitors versus other glucose-lowering drugs: The CVD-Real study (comparative effectiveness of cardiovascular outcomes in new users of sodium-glucose cotransporter-2 inhibitors) Circulation. 2017;136 (3):249-59. doi: 10.1161/ CIRCULATIONAHA.117.029190

27. Gallwitz B. The cardiovascular benefits associated with the use of sodium-glucose cotransporter 2 inhibitors - real-world data. Baptist. Eur Endocrinol 2018;14(1):17-23. doi: 10.17925/EE.2018.14.1.17

28. Koch EAT, Nakhoul R, Nakhoul F, Nakhoul N. Autophagy in diabetic nephropathy: a review. Int Urol Nephrol. 2020;52(9):1705-1712. doi: 10.1007/ s11255-020-02545-4
29. Hallow KM, Helmlinger G, Greasley PJ, McMurray JJV, Boulton DW. Why do SGLT2 inhibitors reduce heart failure hospitalization? A differential volume regulation hypothesis. Diabetes Obes Metab. 2018;20(3):479-487. doi: 10.1111/dom.13126

30. Flores E, Santos-Gallego CG, Diaz-Mejía N, Badimon JJ. Do the SGLT-2 inhibitors offer more than hypoglycemic activity? Eduardo Flores Cardiovasc Drugs Ther. 2018 Apr;32(2):213-222. doi: $10.1007 / \mathrm{s} 10557-018-6786-\mathrm{x}$

31. Boorsma EM, Ter Maaten JM, Damman K, Dinh W, Gustafsson F, Goldsmith S, et al. Congestion in heart failure:a contemporary look at physiology, diagnosis and treatment. Nat Rev Cardiol. 2020;17(10):641-655. doi: 10.1038/s41569-0200379-7

32. Scheen A. Sodium-glucose cotransporter type 2 inhibitors for the treatment of type 2 diabetes mellitus. Nat Rev Endocrinol. 2020;16(10):556-577. doi.org/10.1038/ s41574-020-0392-2

33. Cowie MR, Fisher M. SGLT2 inhibitors: mechanisms of cardiovascular benefit beyond glycemic control. Martin R. Nat Rev Cardiol. 2020;17(12):761-72. doi: 10.1038/s41569-020-0406-8

34. Packer M. SGLT2 inhibitors produce cardiorenal benefits by promoting adaptive cellular reprogramming to induce a state of fasting mimicry: a paradigm shift in understanding their mechanism of action. Diabetes Care. 2020; 43(3): 508-11. doi: 10.2337/ dci19-0074

35. Brito D, Bettencourt P, Carvalho D, Ferreira J, Fontes-Carvalho R, Franco F, et al. Sodium-glucose co-transporter 2 inhibitors in the failing heart: a growing potential. Cardiovasc Drugs Ther. 2020; 34(3):419-36. doi: 10.1007/s10557-020-06973-3

36. Kashiwagi A, Araki S, Maegawa H. Sodium-glucose cotransporter 2 inhibitors represent a paradigm shift in the prevention of heart failure in type 2 diabetes patients. J Diabetes Investig. 2021; 12(1):6-20. doi: $10.1111 /$ jdi.13329

37. Wojcik C, Warden BA. Mechanisms and evidence for heart failure benefits from SGLT2 inhibitors Cezary Wojcik, et al. Curr Cardiol Rep. 2019;21 (10):130. doi: 10.1007/s11886-019-1219-4

38. García-Ropero Á, Vargas-Delgado AP, Santos-Gallego CG, Badimon JJ. Inhibition of sodium glucose cotransporters improves cardiac performance. Int J Mol Sci. 2019;20(13):3289. doi:10. 3390/ijms20133289

39. De Pascalis A, Cianciolo G, Capelli I, Brunori G, La Manna G. SGLT2 inhibitors, sodium and off $]$ target effects: an overview. J Nephrol. 2021;34(3):673-80. doi: 10.1007/s40620-020-00845-7

40. McCullough PA, Kluger AY, Tecson KM, Barbin CM, Lee AY, Lerma EV, et al. Inhibition of the 
sodium-proton antiporter (exchanger) is a plausible mechanism of potential benefit and harm for drugs designed to block sodium glucose co-transporter 2 . Rev Cardiovasc Med. 2018;19(2):51-63. doi:10. 31083/j.rcm.2018.02.021

41. Chung YJ, Park KC, Tokar S, Eykyn TR, Fuller W, Pavlovic D, et al. Off-target effects of SGLT2 blockers: empagliflozin does not inhibit $\mathrm{Na}+\mathrm{H}+\mathrm{ex}-$ changer- 1 or lower $[\mathrm{Na}+] \mathrm{i}$ in the heart. Cardiovas Res. 2020;cvaa323. doi: 10.1093/cvr/cvaa323

42. Kolwicz SC, Purohit S, Tian R. Cardiac Metabolism and Its Interactions with Contraction, Growth, and Survival of the Cardiomyocte. Circ Res. 2013;113(5):603-16. doi:10.1161/CIRCRESAHA. 113.302095

43. Thomas MC, Cherney DZI. The actions of SGLT2 inhibitors on metabolism, renal function and blood pressure. Diabetologia. 2018;61(10):2098-107. doi: 10.1007/s00125-018-4669-0.

44. Schulze PC, Wu JMF. Ketone bodies for the starving heart. Nat Metab. 2020;2(11):1183-5. doi: 10.1038/s42255-020-00310-6

45. Garcia-Ropero A, Santos-Gallego CG, Zafar MU, Badimon JJ. Metabolism of the failing heart and the impact of SGLT2 inhibitors. Expert Opin Drug Metab Toxicol. 2019;15(4):275-85. doi:10.1080/ 17425255.2019.1588886

46. Neubauer S, Horn M, Cramer M, Harre K, Newell JB, Peters W, et al. Myocardial phosphocreatine-to-ATP ratio is a predictor of mortality in patients with dilated cardiomyopathy. Circulation. 1997;96(7):2190-6. doi: 10.1161/01.cir.96.7.2190

47. Santos-Gallego CG, Requena-Ibanez JA, San Antonio R, Ishikawa K, Watanabe S, Picatoste B, et al. Empagliflozin ameliorates adverse left ventricular remodeling in nondiabetic heart failure by enhancing myocardial energetics. J Am Coll Cardiol. 2019;73(15):1931-44. doi: 10.1016/j.jacc.2019.01.056

48. Santos-Gallego CG, Vargas-Delgado AP, Requena-Ibanez JA, Garcia-Ropero A, Mancini D, Pinney S, et al. Randomized trial of empagliflozin in non-diabetic patients with heart failure and reduced ejection fraction. J Am Coll Cardiol. 2021;77(3): 243-55. doi: 10.1016/j.jacc.2020.11.008

49. Avogaro A, Fadini GP, Del Prato S. Reinterpreting cardiorenal protection of renal sodium-glucose cotransporter 2 inhibitors via cellular life history programming. Diabetes Care. 2020;43(3):501-7. doi: $10.2337 / \mathrm{dc} 19-1410$
50. Packer M. Reconceptualization of the molecular mechanism by which sodium-glucose cotransporter 2 inhibitors reduce the risk of heart failure events. Circulation. 2019;140(6):443-5. doi: 10.1161/CIRCULATIONAHA.119.040909

51. Chen ZZ, Wang JY, Kang Y, Yang QY, Gu XY, Zhi DL, et al. Anti-inflammatory effects of sodium-glucose co-transpoter 2 inhibitors on atherosclerosis. Kang Y. Vascul Pharmacol. 2020; 133-34:106779. doi: 10.1016/j.vph.2020.106779

52. Lytvyn Y, Bjornstad P, Udell JA, Lovshin JA, Cherney DZI. Sodium glucose cotransporter-2 inhibition in heart failure: potential mechanisms, clinical applications, and summary of clinical trials. Circulation. 2017;136(17):1643-58. doi: 10.1161/CIRCULATIONAHA.117.030012

53. Garcia-Ropero A, Santos-Gallego CG, Zafar MU, Badimon JJ. Metabolism of the failing heart and the impact of SGLT2 inhibitors. Expert Opin Drug Metab Toxicol. 2019 Apr;15(4):275-85. doi: 10.1080/17425255.2019.1588886

54. Santos-Gallego CG, Requena Ibáñez JA, San Antonio R, Ishikawa K, Watanabe S, Picatoste Botija MB, et al. Empagliflozin induces a myocardial metabolic shift from glucose consumption to ketone metabolism that mitigates adverse cardiac remodeling and improves myocardial contractility. J Am Coll Cardiol. 2018;71(11 Supl):A674.

55. Bailey CJ. Uric acid and the cardio-renal effects of SGLT2 inhibitors Clifford J. Diabetes Obes Metab. 2019;21(6):1291-8. doi: 10.1111/dom.13670

56. Das SR, Everett BM, Birtcher KK, Brown JM, Januzzi JL, Kayani RR, et al. 2020 Expert consensus decision pathway on novel therapies for cardiovascular risk reduction in patients with type 2 diabetes. a report of the American College of Cardiology Solution Set Oversight Committee. J Am Coll Cardiol. 2020;76(9):1117-45. doi.org/10.1016/j.jacc. 2020.05.037

57. Marx N, Davies MJ, Grant PJ, Mathieu C, Petrie JR, Cosentino F, et al. Guideline recommendations and the positioning of newer drugs in type 2 diabetes care. Lancet Diabetes Endocrinol. 2021;9(1): 46-52. doi:10.1016/S2213-8587(20) 30343-0. Fe de errata: Lancet Diabetes Endocrinol. 2021;9(1):e1.

58. Neuen BL, Jardine MJ, Perkovic V. Sodium-glucose cotransporter 2 inhibition: which patient with chronic kidney disease should be treated in the future? Nephrol Dial Transplant. 2020;35(Supl1):i48-55. doi: $10.1093 / \mathrm{ndt} / \mathrm{gfz} 252$ 